\title{
On the classification of certain fusion categories
}

\author{
David Jordan and Eric Larson
}

\begin{abstract}
We advance the classification of fusion categories in two directions. Firstly, we completely classify integral fusion categories - and consequently, semi-simple Hopf algebras - of dimension $p q^{2}$, where $p$ and $q$ are distinct primes. This case is especially interesting because it is the simplest class of dimensions where not all integral fusion categories are grouptheoretical. Secondly, we classify a certain family of $\mathbb{Z} / 3 \mathbb{Z}$-graded fusion categories, which are generalizations of the $\mathbb{Z} / 2 \mathbb{Z}$-graded Tambara-Yamagami categories. Our proofs are based on the recently developed theory of extensions of fusion categories.
\end{abstract}

Mathematics Subject Classification (2000). Primary 18D10; Secondary 20 J99.

Keywords. Fusion categories, Tambara-Yamagami categories, finite dimensional Hopf algebras.

\section{Introduction and results}

Recall that a fusion category is a semi-simple rigid monoidal category with finitely many simple objects $X_{i}$, with simple unit $X_{0}=\mathbf{1}$, such that $\operatorname{End}\left(X_{i}\right)=\mathbb{C}$ for all $i$. The goal of this article is to obtain two classification results for fusion categories, and to apply one of them to the classification of semi-simple Hopf algebras. We begin by explaining how the main results of the article fit into the existing literature.

Classification of fusion categories is an important and difficult problem. More specifically, by the Ocneanu rigidity theorem (see [ENO1]), there are finitely many fusion categories of a given dimension and, in particular, finitely many fusion categories with a given fusion ring $R$ (called categorifications of $R$ ). This leads to the natural problems of classifying all fusion categories of a given dimension and categorifications of a given fusion ring. In full generality these problems are very hard; for example, the first problem includes the classification of finite groups and Lie groups. However, for certain dimensions and certain fusion rings these problems are sometimes tractable and lead to interesting results. For example, there exist classifications of fusion categories of dimension $p, p^{2}$ (see [ENO1]), $p q$ ([EGO]) or $p q r$ ([ENO2]), where $p, q, r$ are distinct primes. In [TY] categorifications of Tambara-Yamagami rings are classified. In addition, there is a simple description of group-theoretical cat- 
egories, i.e., categories that are Morita equivalent to a pointed category (see [ENO1]) and all categories whose dimension is a prime power are of this type (see [DGNO]).

In this article we extend these classification results in two directions. Our first result is the classification of integral fusion categories of dimension $p q^{2}$, where $p$ and $q$ are distinct primes. This case is interesting because it is the first class of dimensions for which an integral fusion category need not be group-theoretical, and significantly new methods are needed to get a classification. ${ }^{1}$ The second result is the classification of categorifications of certain fusion rings $R_{p, G}$, associated with a finite group $G$. These rings are generalizations of the Tambara-Yamagami rings (which correspond to $p=2$ ). Namely, we obtain a complete classification of categorifications of such rings for $p=3$, when the order of the group $G$ is not divisible by 3 . Our main results are Theorems 1.1 and 1.4 below.

Theorem 1.1. Let $p$ and $q$ be primes, and $C$ be an integral ${ }^{2}$ fusion category of Frobenius-Perron dimension $\mathrm{pq}^{2}$. Then exactly one of the following is true:

- $C$ is a group-theoretical category.

- $p=2$, and $\mathcal{C}$ is a Tambara-Yamagami category [TY] corresponding to an anisotropic quadratic form ${ }^{3}$ on $(\mathbb{Z} / q \mathbb{Z})^{2}$; there are two equivalence classes of such categories.

- The prime $p$ is odd and divides $q+1$, and $C$ is one of the categories $e\left(p, q,\left\{\zeta_{1}, \zeta_{2}\right\}, \xi\right)$ we shall explicitly construct. Here, $\zeta_{1} \neq \zeta_{2} \in \mathbb{F}_{q^{2}}$ are such that $\zeta_{1}^{p}=\zeta_{2}^{p}=1$, but $\zeta_{1} \zeta_{2} \neq 1$, and $\xi \in \mathrm{H}^{3}\left(\mathbb{Z} / p \mathbb{Z}, \mathbb{C}^{*}\right) \cong \mathbb{Z} / p \mathbb{Z}$. There are $\left(p^{2}-p\right) / 2$ equivalence classes of such categories.

Corollary 1.2. All semi-simple Hopfalgebras of dimension $p^{2}$ are group-theoretical.

Remark. Another proof of Corollary 1.2, based on different methods, is given in [ENO2].

Our second theorem concerns categorifications of a certain fusion ring $R_{p, G}$ attached to a finite group $G$ and a prime $p$.

Definition 1.3. Let $G$ be a finite group whose order is a square ${ }^{4}$ and let $p \in \mathbb{N}$. Then the fusion ring $R_{p, G}$ is the ring generated by the group ring $\mathbb{Z}[G]$ and $X_{1}, \ldots, X_{p-1}$,

\footnotetext{
${ }^{1}$ All integral fusion categories of dimension $p q r$ classified in a recent preprint [ENO2] are grouptheoretical and thus the techniques of [ENO2] do not work in our situation.

${ }^{2}$ If $p$ and $q$ are odd, then the assumption that $\mathcal{C}$ is integral is redundant.

${ }^{3}$ I.e., of the form $x^{2}-a y^{2}$, where $a$ is a quadratic non-residue.

${ }^{4}$ This assumption is unnecessary when $p=1$ or $p=2$.
} 
with relations

$$
\begin{gathered}
g \otimes X_{i}=X_{i} \otimes g=X_{i}, \quad X_{i}^{*}=X_{p-i}, \\
X_{i} \otimes X_{j}= \begin{cases}\sqrt{|G|} X_{i+j} & \text { if } i+j \neq p, \\
\sum_{g \in G} g & \text { if } i+j=p .\end{cases}
\end{gathered}
$$

Theorem 1.4. Let $A$ be a finite group of order not divisible by 3. Then the fusion ring $R_{3, A}$ admits categorifications if and only if $A$ is abelian, of the form $A \cong \bigoplus_{i=1}^{N}\left(\mathbb{Z} / p_{i}^{n_{i}} \mathbb{Z}\right)^{a_{i}}$, where $p_{i}$ are primes, pairs $\left(p_{i}, n_{i}\right)$ are distinct, and $a_{i}$ are even integers. In this case, there are $3 \prod_{i}\left(\frac{a_{i}}{2}+1\right)$ categorifications.

The proofs of these theorems are based on the solvability of fusion categories of dimension $p^{a} q^{b}$ proved in [ENO2], the new theory of extensions of fusion categories developed in [ENO3], and some intricate linear algebra over finite fields.

The organization of this article is as follows. Section 2 contains a review of standard definitions, and also results from recent literature which we will need. In Section 3, we exhibit non-trivial gradings on the fusion categories of study, and analyze these gradings with the methods from [ENO3]. Sections 4 and 5 present the proofs of Theorem 1.1 and Corollary 1.2, respectively. In Section 6, we focus on the case of $\mathbb{Z} / 3 \mathbb{Z}$-graded extensions of $\mathrm{Vec}_{A}$. In Section 7 we prove Theorem 1.4.

Remark. It is not difficult to extend Theorem 1.1 to classify non-integral categories of dimension $p q^{2}$. This is because the dimensions of all objects in such a category are necessarily square roots of positive integers. This forces a $\mathbb{Z} / 2 \mathbb{Z}$-grading on the category, which means either $p$ or $q$ is 2 . Then a case by case analysis yields a complete list. We have not included these computations as they are not of particular interest.

Remark. It is also possible to extend Lemmas 6.1 and 6.2, and thus Theorem 1.4, to the situation where $A$ contains no elements of order 9 (i.e., the 3-component of $A$ is a vector space over $\mathbb{F}_{3}$ ). However, it seems that our methods break down if $A$ has a more complicated 3-component.

Remark. Some possible directions of future research would be a generalization of Theorem 1.1 to categories of dimension $p q^{n}, n \geq 3$, and of Theorem 1.4 to $p>3$. These problems reduce to describing orbits of actions of certain reductive subgroups of $O\left(2 n, \mathbb{F}_{q}\right)$ on the Lagrangian Grassmanian. While in general these problems may be intractable, we think that under reasonable simplifying assumptions one can get manageable and interesting classifications.

Acknowledgements. The authors would like to warmly thank Pavel Etingof and Victor Ostrik for posing the problem, and for many helpful conversations as the work 
progressed. We are grateful to Victor Ostrik for explaining to us how Corollary 1.2 could be easily derived from our results. The work of both authors was supported by the Research Science Institute and conducted in the Department of Mathematics at MIT.

\section{Preliminaries}

In this section we recall several basic notions about fusion categories. For more details see [ENO1], [ENO2], [ENO3], [O1]. For the remainder of the article, $\mathcal{C}$ and $\mathscr{D}$ are fusion categories, $G$ is any finite group, and $A$ is a finite abelian group.

Definition 2.1. A fusion (or based) ring $R$ is an associative ring which is free of finite rank as a $\mathbb{Z}$-module, with fixed $\mathbb{Z}$-basis $B=\left\{X_{i}\right\}$ containing $X_{0}=\mathbf{1}$, and an involution ${ }^{*}: B \rightarrow B$ extending to an anti-involution $R \rightarrow R$ such that:

(i) $X_{i} X_{j}=\sum N(i, j, k) X_{k}$ for all $i, j$, where $N(i, j, k)$ are non-negative integers,

(ii) $N\left(i, j^{*}, 0\right)=\delta_{i j}$.

Definition 2.2. The fusion ring of $\mathcal{C}$, denoted $K(\mathcal{C})$, has as its basis the isomorphism classes of simple objects of $\mathcal{C}$, with $N(i, j, k)$ equal to the multiplicity of $X_{k}$ in $X_{i} \otimes X_{j}$, and ${ }^{*}$ defined by the duality in $\mathcal{C}$. A categorification of a fusion ring $R$ is a fusion category $\mathcal{C}$ such that $K(\mathcal{C})=R$.

A fusion ring can have more than one categorification, or none at all. For example, consider the group ring $\mathbb{Z}[G]$ of a finite group $G$ (with basis $\{g \in G\}$ ). Categorifications of these rings are known as pointed categories. One such categorification is the category of $G$-graded vector spaces, $V=\bigoplus_{g \in G} V_{g}$, with the trivial associativity isomorphism. We can construct other categorifications by letting the associativity isomorphism $\alpha$ be defined on the graded components by

$$
\left(U_{g} \otimes V_{h}\right) \otimes W_{k} \stackrel{\xi(g, h, k)}{\longrightarrow} U_{g} \otimes\left(V_{h} \otimes W_{k}\right),
$$

for some 3-cocycle $\xi \in Z^{3}\left(G, \mathbb{C}^{*}\right)$. We denote the resulting category $\operatorname{Vec}_{G, \xi}$ (or just $\operatorname{Vec}_{G}$ if $\xi$ is trivial). It is well known that $\operatorname{Vec}_{G, \xi}$ depends only on the cohomology class of $\xi$, and these categories are the only pointed categories. Thus, categorifications of $\mathbb{Z}[G]$ are parameterized by the $\operatorname{set}^{3}\left(G, \mathbb{C}^{*}\right) / \operatorname{Aut}(G)$. On the other hand, the twodimensional fusion ring with the basis $\{\mathbf{1}, X\}$ and the fusion rules $X^{2}=\mathbf{1}+n X$ has two categorifications when $n=0,1$, and no categorifications for $n>1$ (see [O3]).

Definition 2.3. The Frobenius-Perron dimension FPdim $X_{i}$ of $X_{i}$ is the largest positive eigenvalue of the matrix $N_{i}$ with entries $N(i, j, k)$ (such an eigenvalue exists by the Frobenius-Perron theorem). 
For categories of group representations (or more generally, representations of a semi-simple quasi-Hopf algebra), this is the vector space dimension; however, in general the dimension need not be an integer - it is only an algebraic integer. If all FPdim $X_{i}$ are integers, we call the category integral.

Definition 2.4. The dimension $|\mathcal{C}|$ of $\mathcal{C}$ is the sum of the squares of dimensions of all simple objects of $\mathcal{C}$.

For the category of representations of a semi-simple quasi-Hopf algebra $H,|\mathcal{C}|$ is the vector space dimension of $H$, by Maschke's theorem.

Definition 2.5. We say that $\mathcal{C}$ is graded by $G$ if $\mathcal{C}$ decomposes as a direct sum $\ell=\bigoplus_{g \in G} \ell_{g}$, such that $\ell_{g} \otimes \ell_{h} \subseteq \ell_{g h}$. Let us denote the trivial component of the grading $\ell_{e}$. When $G$ is abelian, we refer to the trivial component as $\ell_{0}$. By an extension of $\mathscr{D}$ by $G$, we mean a $G$-graded fusion category $\ell$ with $\ell_{e}=\mathscr{D}$.

Lemma 2.6 ([O2]). Let $\mathcal{C}$ be a G-graded fusion category whose trivial component $\ell_{e}$ is pointed, with some component $\mathcal{C}_{g}$ containing a unique simple object. Then $\ell_{e} \cong \mathrm{Vec}_{A}$, with A abelian.

Proof. The category $\mathcal{C}_{g}$ defines a fiber functor on $\ell_{e}$, which implies that $\ell_{e}=\operatorname{Rep} H$ for some commutative Hopf algebra $H$. Thus, $\xi=0, H=\operatorname{Fun}(G)$. Now, $\operatorname{Rep} H^{*}$ is the dual category to $\ell_{e}$ with respect to $\ell_{g}$, which is the same as $\ell_{e}$. Thus $H^{*}$ is commutative and $G$ is abelian.

Definition 2.7. The Picard group of $\mathcal{C}$, denoted Pic( $(\mathcal{C})$, is the set of all equivalence classes of invertible $\mathcal{C}$-bimodule categories under the operation of the tensor product. Thus, $\operatorname{Pic}(\mathcal{C})$ is the group of equivalence classes of Morita auto-equivalences of $\mathcal{C}$.

Definition 2.8. Two fusion categories $\ell_{\text {and }} \mathcal{C}^{\prime}$ are equivalent, if there is an invertible tensor functor: $\ell \rightarrow \ell^{\prime}$. If we have two categories $\mathcal{C}$ and $\ell^{\prime}$ graded by the same group $G$, then we say that they are grading-equivalent if there is some invertible tensor functor: $\mathcal{C} \rightarrow \ell^{\prime}$ which restricts to a functor $\ell_{g} \rightarrow \ell_{g}^{\prime}$ for each $g \in G$.

Theorem 2.9 ([ENO3]). $\operatorname{Pic}\left(\operatorname{Vec}_{A}\right)$ is the split orthogonal group $O\left(A \oplus A^{*}\right)$.

For completeness, let us sketch a proof. The key point is that Morita equivalences between fusion categories $\mathcal{C}$ and $\mathcal{D}$ are in bijection with braided equivalences between their Drinfeld centers $Z(\mathscr{C})$ and $Z(\mathscr{D})$, and this equivalence maps tensor product of bimodule categories to composition of functors. (See [ENO2], Theorem 3.1.) In particular, the group of Morita auto-equivalences of $\mathcal{C}$ is naturally isomorphic to

\footnotetext{
${ }^{5} \mathrm{By}$ an invertible functor, we mean a functor with a quasi-inverse.
} 
the group of braided auto-equivalences of $Z(\mathcal{C})$. In the case $\ell=\operatorname{Vec}_{A}, Z(\mathcal{C})$ is $\operatorname{Vec}_{A \oplus A^{*}}$, with braiding given by the standard (split) quadratic form. Thus, the group of braided auto-equivalences of $Z(\mathcal{C})$ is isomorphic to $O\left(A \oplus A^{*}\right)$, and the result follows.

Theorem 2.10 ([ENO3]). Fix a fusion category $\mathcal{C}_{e}$. Then categories $\mathcal{C}$, graded by $G$, with trivial component $\mathcal{C}_{e}$ are classified, up to grading-equivalence, by triples $(\rho, h, k)$, where $\rho: G \rightarrow \operatorname{Pic}\left(\ell_{e}\right)$ is a homomorphism, $h \in \mathrm{H}^{2}\left(G, \operatorname{Inv}\left(Z\left(\ell_{e}\right)\right)\right)$, and $k \in \mathrm{H}^{3}\left(G, \mathbb{C}^{*}\right){ }^{6}$ There are obstructions $\phi_{1}(\rho) \in \mathrm{H}^{3}\left(G, \operatorname{Inv}\left(Z\left(\bigodot_{e}\right)\right)\right)$ and $\phi_{2}(\rho, h) \in \mathrm{H}^{4}\left(G, \mathbb{C}^{*}\right)$ which must vanish for $\mathcal{C}(\rho, h, k)$ to exist. Here, we consider this data up to the action of the group of tensor auto-equivalences of $\mathcal{C}_{e}$.

\section{Cyclic extensions of $\mathrm{Vec}_{A}$}

Let us fix primes $p$ and $q$, and a generator $\epsilon$ of $\mathbb{Z} / p \mathbb{Z}$.

Proposition 3.1. Let $|A|$ be coprime to $p$. Then categorifications of $R_{p, A}$ are parameterized by the data $(\rho, \xi)$, where $\xi \in \mathrm{H}^{3}\left(\mathbb{Z} / p \mathbb{Z}, \mathbb{C}^{*}\right) \simeq \mathbb{Z} / p \mathbb{Z}$, and $\rho: \mathbb{Z} / p \mathbb{Z} \rightarrow O\left(A \oplus A^{*}\right)$ is a homomorphism such that if we write

$$
\rho(i)=\left(\begin{array}{ll}
\alpha_{i} & \beta_{i} \\
\gamma_{i} & \delta_{i}
\end{array}\right)
$$

where $\alpha_{i}: A \rightarrow A, \beta_{i}: A^{*} \rightarrow A, \gamma_{i}: A \rightarrow A^{*}, \delta_{i}: A^{*} \rightarrow A^{*}$, then $\beta_{i}$ is an isomorphism for all $i \neq 0$. Two such categorifications are equivalent if and only if they are related by the natural action of $\operatorname{Aut}(\mathbb{Z} / p \mathbb{Z})$ and the subgroup of the orthogonal group of elements of the form

$$
\boldsymbol{v}=\left(\begin{array}{cc}
\psi & 0 \\
\varphi & \psi^{-1 *}
\end{array}\right)
$$

where $\psi^{*} \varphi$ is skew-symmetric.

Proof. Clearly, any categorification $\mathcal{C}$ of the fusion ring $R_{p, A}$ is $\mathbb{Z} / p \mathbb{Z}$-graded. From Lemma 2.6, $\mathcal{C}_{0}=\operatorname{Vec}_{A}$. We must have that $\beta_{i}$ is an isomorphism for all $i \neq 0$ since $\bigodot_{i}=\left\langle X_{i}\right\rangle$, and $\operatorname{FPdim}\left(X_{i}\right)^{2}=|A|=\left|\operatorname{Im} \beta_{i}\right|$. Now, $\left|\operatorname{Inv}\left(Z\left(C_{0}\right)\right)\right| \operatorname{divides}\left|Z\left(\complement_{0}\right)\right|$, since the dimension of any subcategory divides the dimension of the category. But, $\left|Z\left(\mathscr{C}_{0}\right)\right|=\left|\mathcal{C}_{0}\right|^{2}$. Because we are assuming that $p$ is coprime to $|A|, p$ is coprime to $\left|\operatorname{Inv}\left(Z\left(\mathcal{C}_{0}\right)\right)\right|$, whereby $\mathrm{H}^{*}\left(\mathbb{Z} / p \mathbb{Z} \operatorname{Inv}\left(Z\left(\mathcal{C}_{0}\right)\right)\right)=0$. (In particular this implies

\footnotetext{
${ }^{6}$ Actually the data $h$ and $k$ belong to torsors over the groups $\mathrm{H}^{2}\left(G, \operatorname{Inv}\left(Z\left(\mathcal{C}_{e}\right)\right)\right)$ and $\mathrm{H}^{3}\left(G, \mathbb{C}^{*}\right)$, respectively, rather than to the groups themselves. This is a technical point which is not going to matter for our considerations. Here $\operatorname{Inv}(\mathscr{D})$ denotes the group of invertible objects of $\mathscr{D}$.
} 
that the third cohomology group is trivial.) We also have no choice for the second piece of data, since the second cohomology group is also trivial. Finally, the second obstruction vanishes because $\mathrm{H}^{4}\left(\mathbb{Z} / p \mathbb{Z}, \mathbb{C}^{*}\right)=0$. Therefore, by [ENO3], such categories are determined up to grading-equivalence by the data $(\rho, \xi)$.

It is clear that if $\ell^{\prime}, \ell^{\prime \prime}$ are two categorifications of $R_{p, A}$, then any equivalence between $\ell^{\prime}$ and $\ell^{\prime \prime}$, will preserve the grading, up to the action of $\operatorname{Aut}(\mathbb{Z} / p \mathbb{Z})$. Thus, since the subgroup of the orthogonal group which acts on the data $(\rho, \xi)$ is the group of auto-equivalences of $\ell_{0}=\mathrm{Vec}_{A}$, we conclude the statement of this proposition.

Now we consider what happens when instead of requiring that each graded component $\ell_{g}(g \neq 0)$ contains a unique simple object, we only require that the graded component $\ell_{\epsilon}$ contains a unique simple object. Since this condition is not invariant under the action of $\operatorname{Aut}(\mathbb{Z} / p \mathbb{Z})$, we classify these categorifications up to gradingequivalence.

Theorem 3.2. Let $p \in \mathbb{N}$ be relatively prime to $|A|$. Then $\mathbb{Z} / p \mathbb{Z}$-graded categories $\mathcal{C}_{\text {with trivial component }} \operatorname{Vec}_{A}$ such that $\mathcal{C}_{\epsilon}$ contains a unique simple object are parameterized up to grading-equivalence by an element of $\mathrm{H}^{3}\left(\mathbb{Z} / p \mathbb{Z}, \mathbb{C}^{*}\right) \simeq \mathbb{Z} / p \mathbb{Z}$, together with a map $\alpha: A \rightarrow A$ and an isomorphism $\gamma: A \rightarrow A^{*}$ such that $\gamma^{*} \alpha$ is skew-symmetric and

$$
\left(\begin{array}{cc}
\alpha & \mathrm{Id} \\
\left(\gamma^{-1} \gamma^{*}\right)^{-1} & 0
\end{array}\right)^{p}=\mathrm{Id}
$$

Proof. As in Proposition 3.1, our categories are determined by the data $(\rho, \xi)$, except that we only require $\beta_{1}$ to be an isomorphism.

To specify the homomorphism $\rho$, it suffices to give the image of the generator $\rho(\epsilon)$, say

$$
\boldsymbol{M}=\left(\begin{array}{cc}
\alpha & \beta \\
\gamma & \delta
\end{array}\right) \in O\left(A \oplus A^{*}\right)
$$

such that $\beta$ is invertible and $\boldsymbol{M}^{p}=\mathrm{Id}$. However, we must consider such matrices $\boldsymbol{M}$ up to conjugation by elements of the form

$$
\left(\begin{array}{cc}
\text { Id } & 0 \\
\varphi & \text { Id }
\end{array}\right)
$$

where $\varphi$ is skew-symmetric, since conjugation by elements of the form

$$
\left(\begin{array}{cc}
\psi & 0 \\
0 & \psi^{*-1}
\end{array}\right)
$$

amounts to the change of basis $(\alpha, \gamma) \mapsto\left(\psi^{-1} \alpha \psi, \psi^{*} \gamma \psi\right)$.

Claim 3.3. For a given matrix in the form (2), there is exactly one matrix of the form (3) which conjugates it into a matrix where $\delta=0$. 
Proof. Observe that when we conjugate (2) by (3) we obtain

$$
\left(\begin{array}{cc}
\text { Id } & 0 \\
\varphi & \text { Id }
\end{array}\right)\left(\begin{array}{cc}
\alpha & \beta \\
\gamma & \delta
\end{array}\right)\left(\begin{array}{cc}
\text { Id } & 0 \\
-\varphi & \text { Id }
\end{array}\right)=\left(\begin{array}{cc}
\alpha-\beta \varphi & \beta \\
\star & \varphi \beta+\delta
\end{array}\right) .
$$

So a matrix in the form of (3) conjugates $\boldsymbol{M}$ into a matrix where $\delta=0$ if and only if $\varphi=-\delta \beta^{-1}=-\beta^{-1 *} \beta^{*} \delta \beta^{-1}$, which is skew-symmetric because $\boldsymbol{M} \in O\left(A \oplus A^{*}\right)$. form

Thus, we have reduced the problem to classifying the set of all matrices $\boldsymbol{M}$ in the

$$
\left(\begin{array}{ll}
\alpha & \beta \\
\gamma & 0
\end{array}\right) \in O\left(A \oplus A^{*}\right)
$$

whose $p$-th power is the identity matrix. The condition that $M \in O\left(A \oplus A^{*}\right)$ can be expressed as $\gamma^{*} \alpha$ being skew-symmetric, and $\beta=\gamma^{*-1}$. Therefore, we want to find linear maps $\alpha, \gamma$, with $\gamma$ invertible, such that $\gamma^{*} \alpha$ is skew-symmetric and

$$
\left(\begin{array}{cc}
\alpha & \gamma^{*-1} \\
\gamma & 0
\end{array}\right)^{p}=\mathrm{Id}
$$

Now, conjugating $\boldsymbol{M}$ by anything in the general linear group does not change the property that $\boldsymbol{M}^{p}=\mathrm{Id}$, so we can replace $\boldsymbol{M}$ with

$$
\left(\begin{array}{cc}
\mathrm{Id} & 0 \\
0 & \gamma^{*-1}
\end{array}\right)\left(\begin{array}{cc}
\alpha & \gamma^{*-1} \\
\gamma & 0
\end{array}\right)\left(\begin{array}{cc}
\mathrm{Id} & 0 \\
0 & \gamma^{*}
\end{array}\right)=\left(\begin{array}{cc}
\alpha & \mathrm{Id} \\
\left(\gamma^{-1} \gamma^{*}\right)^{-1} & 0
\end{array}\right)
$$

Therefore (4) may be replaced with the condition (1).

Remark. One may easily deduce Theorem 3.2 of [TY] as a corollary to Theorem 3.2 above.

Lemma 3.4. Let $C$ be a cyclic $q$-group. Then, up to equivalence, there is exactly one non-degenerate skew-symmetric map $\gamma: C \oplus C \rightarrow(C \oplus C)^{*}$.

Proof. Identical to the proof of the corresponding theorem for vector spaces.

Lemma 3.5. Let $a \in \mathbb{Z}$ such that $q \nmid a^{2}-4$, and let $C$ be a cyclic $q$-group. Then, up to equivalence, there is exactly one isomorphism $\gamma: C \oplus C \rightarrow(C \oplus C)^{*}$ such that $\left(\gamma^{-1} \gamma^{*}\right)^{2}=a \gamma^{-1} \gamma^{*}-$ Id.

Proof. Write $C=\mathbb{Z} / q^{n} \mathbb{Z}$. First, assume to the contrary that $q^{n-1} \gamma^{-1} \gamma^{*}$ is multiplication by some constant $q^{n-1} \lambda$. Then $q^{n-1} \gamma=\left(q^{n-1} \gamma^{*}\right)^{*}=\left(q^{n-1} \lambda \gamma\right)^{*}=$ $q^{n-1} \lambda^{2} \gamma$. Thus, modulo $q, \lambda^{2}=1$, so $q^{n-1} \operatorname{Id}=q^{n-1}(\lambda \mathrm{Id})^{2}=q^{n-1}(a \lambda \mathrm{Id}-\mathrm{Id})=$ 
$q^{n-1}(a \lambda-1)$ Id. Thus, $1=\lambda^{2}=(2 / a)^{2}$ modulo $q$, which contradicts our assumption that $q$ does not divide $a^{2}-4$. Thus, the characteristic polynomial of $x=\gamma^{-1} \gamma^{*}$ is $t^{2}-a t+1$, since that is the minimal polynomial and of the correct degree. Now we claim that there is exactly one equivalence class for $x$, namely the class of

$$
x=\left(\begin{array}{cc}
a & 1 \\
-1 & 0
\end{array}\right)
$$

Since the characteristic polynomial of $x$ is $t^{2}-a t+1, x$ has the form

$$
x=\left(\begin{array}{cc}
a-d & b \\
c & d
\end{array}\right),
$$

where $d(a-d)-b c=1$, and $a \neq 2 d$ modulo $q$ if $b=c=0$ modulo $q$, because we already proved that $q^{n-1} x$ was not multiplication by a scalar. If $d(a-d)-b c=1$, it is straight-forward to check

$$
\left(\begin{array}{cc}
a-d & b \\
c & d
\end{array}\right)\left(\begin{array}{cc}
(a-d) y+b & y \\
c y+d & 1
\end{array}\right)=\left(\begin{array}{cc}
(a-d) y+b & y \\
c y+d & 1
\end{array}\right)\left(\begin{array}{cc}
a & 1 \\
-1 & 0
\end{array}\right)
$$

Thus, we are done if there is $y \in \mathbb{F}_{q}$ so that

$$
-c y^{2}+(a-2 d) y+b=\operatorname{det}\left(\begin{array}{cc}
(a-d) y+b & y \\
c y+d & 1
\end{array}\right) \neq 0,
$$

as we can take an arbitrary lift of $y$ into $\mathbb{Z} / q^{n} \mathbb{Z}$ to finish. Since we cannot have $b=c=0, a-2 d=0$ modulo $q$, there is some $y \in \mathbb{F}_{q}$ that finishes the claim, unless $q=2$ and $-c y^{2}+(a-2 d) y+b=y^{2}+y$. But in the latter case, it follows that $\operatorname{det} x=0$, which contradicts the invertibility of $x$. Therefore, we may assume (5). If we write

$$
\gamma=\left(\begin{array}{ll}
b & c \\
d & e
\end{array}\right)
$$

then we have that

$$
\left(\begin{array}{ll}
b & d \\
c & e
\end{array}\right)=\gamma^{*}=\gamma x=\left(\begin{array}{ll}
b & c \\
d & e
\end{array}\right)\left(\begin{array}{cc}
a & 1 \\
-1 & 0
\end{array}\right)=\left(\begin{array}{ll}
a b-c & b \\
a d-e & d
\end{array}\right) .
$$

Therefore,

$$
\gamma=\left(\begin{array}{cc}
d & (a-1) d \\
d & d
\end{array}\right)
$$

But, for any $y, t$, such a map is equivalent to

$$
\left(\begin{array}{cc}
a y+t & -y \\
y & t
\end{array}\right) \gamma\left(\begin{array}{cc}
a y+t & y \\
-y & t
\end{array}\right)=\left(\begin{array}{cc}
d\left(y^{2}+a y t+t^{2}\right) & (a-1) d\left(y^{2}+a y t+t^{2}\right) \\
d\left(y^{2}+a y t+t^{2}\right) & d\left(y^{2}+a y t+t^{2}\right) .
\end{array}\right) .
$$


In order to show the statement of this lemma, it suffices to show that there are $y, t \in$ $\mathbb{Z} / q^{n} \mathbb{Z}$ so that $y^{2}+a y t+t^{2}=1 / d$. By Hensel's Lemma, it suffices to prove there is a solution modulo $q$ such that $2 y+a t \neq 0$.

If $q=2$, this is clear, since we can take $y=t=1$ ( $d$ and $a$ must both be 1 , since $\gamma$ is invertible). If $q \neq 2$, then it is equivalent to $(2 y+a t)^{2}+\left(4-a^{2}\right) t^{2}=4 / d$. Observe that we may choose $2 y+a t$ and $t$ independently; that is we want to find $z, w \in \mathbb{F}_{q}$ so that $z^{2}+\left(4-a^{2}\right) w^{2}=4 / d$. If $4 / d$ is a quadratic residue, we may choose $z^{2}=4 / d, w^{2}=0$. Therefore, suppose that $d$ is not a quadratic residue. Then, if $\left(4-a^{2}\right)$ is also not a quadratic residue, we may take $z=0, w^{2}=4 /\left(\left(4-a^{2}\right) d\right)$. So, say that $4-a^{2}$ is a quadratic residue, $4-a^{2}=f^{2}$. Then our equation becomes $z^{2}+(f w)^{2}=4 / d$, where $f \neq 0$. Define the sets $S_{i}=\{2 / d+i, 2 / d-i\}$ for $i=0,1,2, \ldots, \frac{q-1}{2}$. By the pigeonhole principle, we must either have that $2 / d$ is a quadratic residue, in which case we are done, or that two quadratic residues in the same $S_{i}$, say $\left\{z^{2},(f w)^{2}\right\}=S_{i}$, which implies that $z^{2}+(f w)^{2}=4 / d$.

Lemma 3.6. Let $\mathcal{C}$ be an integral fusion category of Frobenius-Perron dimension $p q^{2}$. Then either $\mathcal{C}$ is faithfully graded by $\mathbb{Z} / p \mathbb{Z}$, or $\mathcal{C}$ is group-theoretical.

Proof. By [ENO2], any fusion category of dimension $p^{m} q^{n}$ is Morita equivalent to a nilpotent fusion category. Therefore, every fusion category of dimension $p q^{2}$ is either Morita equivalent to a category with a faithful $\mathbb{Z} / q \mathbb{Z}$-grading, or one with a faithful $\mathbb{Z} / p \mathbb{Z}$-grading.

Suppose that $\mathcal{C}$ is Morita equivalent to a category $\mathcal{D}$ with a faithful $\mathbb{Z} / q \mathbb{Z}$-grading. Let $\mathscr{D}_{0}$ be the trivial component of the grading. Then $\mathscr{D}_{0}$ is an integral fusion category of dimension $p q$. Therefore, by [EGO], $\mathscr{D}_{0}$ is group-theoretical and thus Morita equivalent to a pointed category $\mathscr{D}_{0}^{\prime}$, and $\mathscr{D}$ is Morita equivalent to some $\mathbb{Z} / q \mathbb{Z}$ graded category $\mathscr{D}^{\prime}$ whose trivial component is $\mathscr{D}_{0}^{\prime}$ (see [ENO2], Lemma 3.3). But the possible dimensions of objects of $\mathscr{D}^{\prime}$ are only $1, \sqrt{q}, \sqrt{p}, \sqrt{q p}$. Thus, since Morita equivalence preserves integrality, $\mathscr{D}^{\prime}$ is pointed, and therefore $\varphi$ is grouptheoretical.

Next suppose that $\mathcal{C}$ is not Morita equivalent to a category $\mathcal{D}$ with a faithful $\mathbb{Z} / q \mathbb{Z}$ grading, and that it does not possess a faithful $\mathbb{Z} / p \mathbb{Z}$-grading. Then, since all fusion categories of dimension $p q^{2}$ are solvable [ENO2], $\ell$ is an equivariantization of some category $\ell_{0}$ of dimension $q^{2}$ by $\mathbb{Z} / p \mathbb{Z}$. Since all integral categories of dimension $q^{2}$ are pointed, and any equivariantization of a pointed category is group-theoretical, the statement of this lemma follows.

Lemma 3.7. Any integral fusion category of Frobenius-Perron dimension $p q^{2}$, which

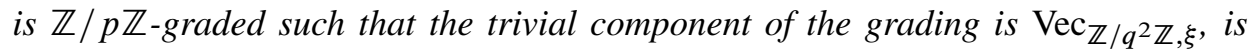
group-theoretical.

Proof. Since our category is integral, it is either pointed, in which case we are done, or there is an object of dimension $q$. If that is the case, then by Lemma $2.6, \xi=0$. Thus, 
the Picard group of the trivial component is $O\left(\mathbb{Z} / q^{2} \mathbb{Z}+\left(\mathbb{Z} / q^{2} \mathbb{Z}\right)^{*}\right)$. Therefore, the category must be group-theoretical, since $q\left(\mathbb{Z} / q^{2} \mathbb{Z}+\left(\mathbb{Z} / q^{2} \mathbb{Z}\right)^{*}\right)$ is an invariant Lagrangian subspace, under any action, with respect to the split quadratic form.

Lemma 3.8. Let $\alpha$ and $\gamma$ be $2 \times 2$ matrices, with $\gamma$ invertible, and $\alpha^{*} \gamma$ skew-symmetric. Then $\alpha$ commutes with $\gamma^{-1} \gamma^{*}$.

Proof. Let $\varphi=\alpha \gamma^{-1}$. Since $\alpha^{*} \gamma$ is skew-symmetric, $\varphi$ is as well. Explicit computation reveals that $\gamma \varphi \gamma^{*}=\gamma^{*} \varphi \gamma$, which implies that $\alpha \gamma^{-1} \gamma^{*}=\gamma^{-1} \gamma^{*} \alpha$.

\section{Proof of Theorem 1.1}

Write $A=(\mathbb{Z} / q \mathbb{Z})^{2}$. By Lemmas 3.6 and 3.7, either $\mathcal{C}$ is group-theoretical, or $\mathcal{C}$ is a $\mathbb{Z} / p \mathbb{Z}$-graded category with trivial component $\operatorname{Vec}_{A, \xi}$. Since $\mathcal{C}$ has all objects of integral dimension by assumption, either $\mathcal{C}$ is pointed, in which case we are done, or $\mathcal{C}$ has an object of dimension $q$, in which case by Lemma $2.6, \xi=0$. Therefore, if $p=2$, by [TY] such categories are parameterized by a quadratic form $\gamma$, and by [GNN] such categories are group-theoretical if and only if there is a subgroup $L \subset A$ such that $|L|=\sqrt{|A|}=q$ and such that $\gamma$ is 0 when restricted to $L$. Since $A$ is a two-dimensional vector space, this is equivalent to the form $\gamma$ being isotropic. This completes the proof if $p=2$. Thus, we will assume that $p$ is odd.

In particular, $\mathcal{C}$ is a $\mathbb{Z} / p \mathbb{Z}$-graded category with trivial component $\operatorname{Vec}_{A}$ such that $\ell_{\epsilon}$ contains a single simple object, where $\epsilon$ is the generator of $\mathbb{Z} / p \mathbb{Z}$. From Theorem 3.2, such $\mathcal{C}$ are parameterized by an element of $\mathrm{H}^{3}\left(\mathbb{Z} / p \mathbb{Z}, \mathbb{C}^{*}\right)$, together with an equivalence class of a pair of maps $\alpha: A \rightarrow A, \gamma: A \rightarrow A^{*}$, where $\gamma$ is an isomorphism, which satisfy $\gamma^{*} \alpha$ skew-symmetric, and (1).

Write $x$ for $\gamma^{-1} \gamma^{*}$. From Lemma 3.8, we have that $\alpha$ and $x$ commute. Consider the matrix

$$
\boldsymbol{M}=\left(\begin{array}{cc}
\alpha & \mathrm{Id} \\
x^{-1} & 0
\end{array}\right)
$$

as a two-by-two matrix over the commutative subring of matrices generated by $\alpha$ and $x$. Then, since $\boldsymbol{M}^{p}=\mathrm{Id}$, we must have $\operatorname{det}(\boldsymbol{M})^{p}=\mathrm{Id}$. Since $\operatorname{det}(\boldsymbol{M})=-x^{-1}$, we have that $x^{p}=-\mathrm{Id}$. Over the algebraic closure of $\mathbb{F}_{q}$, we may choose a basis such that

$$
x=\left(\begin{array}{cc}
-\mu & 0 \\
0 & -\lambda
\end{array}\right)
$$

where $\lambda^{p}=\mu^{p}=1$. Since $\operatorname{det} x=\operatorname{det} \gamma^{-1} \operatorname{det} \gamma^{*}=1$, it follows that $\mu=\lambda^{-1}$. By Lemma 3.5 there is exactly one solution, up to equivalence, to the equation $\gamma^{*}=\gamma x$, where $\gamma$ is invertible. This equation is a system of four linear equations in the entries 
of the matrix for $\gamma$. Solving it yields

$$
\gamma=\left(\begin{array}{cc}
0 & -\lambda^{-1} g \\
g & 0
\end{array}\right)
$$

Write

$$
\alpha=\left(\begin{array}{ll}
a & b \\
c & d
\end{array}\right)
$$

Then we have that

$$
\gamma^{*} \alpha=\left(\begin{array}{cc}
0 & g \\
-\lambda g & 0
\end{array}\right)\left(\begin{array}{ll}
a & b \\
c & d
\end{array}\right)=\left(\begin{array}{cc}
c g & d g \\
-a \lambda^{-1} g & -b \lambda^{-1} g
\end{array}\right)
$$

is skew-symmetric. In other words,

$$
\alpha=\left(\begin{array}{cc}
a & 0 \\
0 & a \lambda^{-1}
\end{array}\right)
$$

We must therefore have

$$
\left(\begin{array}{cccc}
a & 0 & 1 & 0 \\
0 & a \lambda^{-1} & 0 & 1 \\
-\lambda & 0 & 0 & 0 \\
0 & -\lambda^{-1} & 0 & 0
\end{array}\right)^{p}=\mathrm{Id},
$$

or equivalently, there exist $\zeta_{1}, \zeta_{2}$ distinct $p$-th roots of unity such that

$$
\left(\begin{array}{cc}
a & 1 \\
-\lambda & 0
\end{array}\right)
$$

is conjugate to

$$
\left(\begin{array}{cc}
\zeta_{1} & 0 \\
0 & \zeta_{2}
\end{array}\right)
$$

i.e., $a=\zeta_{1}+\zeta_{2}, \lambda=\zeta_{1} \zeta_{2}$. Since $p$ is odd, $x$ and $\alpha$ have the same block form and therefore the same centralizer.

Claim 4.1. There exists a basis so that both $x$ and $\alpha$ are matrices over $\mathbb{F}_{q}$ if and only if $p \mid q^{2}-1$.

Proof. Observe that $p\left|q^{2}-1 \Longleftrightarrow\right| \operatorname{Gal}\left(\mathbb{F}_{q}\left[\zeta_{p}\right]: \mathbb{F}_{q}\right) \mid \leq 2 \Longleftrightarrow \zeta+\zeta^{-1} \in \mathbb{F}_{q}$ for each $\zeta$ which is a primitive $p$-th root of unity. When $\lambda=1, x=-\operatorname{Id}$ and $\alpha=$ $\left(\zeta_{1}+\zeta_{1}^{-1}\right)$ Id are central, and are matrices over $\mathbb{F}_{q} \Longleftrightarrow \zeta_{1}+\zeta_{1}^{-1} \in \mathbb{F}_{q} \Longleftrightarrow p \mid q^{2}-1$. When $\lambda \neq 1$, to see the "only if" part, observe that since $\lambda+\lambda^{-1}=-\operatorname{tr} x \in \mathbb{F}_{q}$, we know that $p \mid q^{2}-1$. To see the "if" part, let

$$
\psi=\left(\begin{array}{cc}
\lambda & -1 \\
-1 & \lambda
\end{array}\right)
$$


and observe that

$$
\begin{aligned}
\psi\left(\gamma^{-1} \gamma^{*}\right) \psi^{-1} & =\left(\begin{array}{cc}
-\left(\lambda+\lambda^{-1}\right) & -1 \\
1 & 0
\end{array}\right), \\
\psi \alpha \psi^{-1} & =\left(\begin{array}{cc}
\left(\lambda+\lambda^{-1}+1\right) \frac{a}{\lambda+1} & \frac{a}{\lambda+1} \\
-\frac{a}{\lambda+1} & \frac{a}{\lambda+1}
\end{array}\right),
\end{aligned}
$$

both of which are matrices over $\mathbb{F}_{q}$.

It is thus clear that if $q^{2}-1$ is not divisible by $p$, there are no non-pointed categorifications, and if $q^{2}-1$ is divisible by $p$, then up to grading-equivalence, nonpointed categorifications are determined by an element of $\mathrm{H}^{3}\left(\mathbb{Z} / p \mathbb{Z}, \mathbb{C}^{*}\right)$ together with unordered pairs $\left\{\zeta_{1}, \zeta_{2}\right\}$ of distinct $p$-th roots of unity under the equivalence $\left\{\zeta_{1}, \zeta_{2}\right\} \sim\left\{\zeta_{1}^{-1}, \zeta_{2}^{-1}\right\}$. Such pairs determine the pair $(\gamma, \alpha)$ uniquely because they determine $\gamma$ uniquely, and $\gamma^{-1} \gamma^{*}$ and $\alpha$ have the same centralizer.

Claim 4.2. The category $\ell$ is group-theoretical if and only if $\zeta_{1} \zeta_{2} \in \mathbb{F}_{q}$.

Proof. The resulting category is group-theoretical if and only if there exists a Lagrangian subspace $L \subset A \oplus A^{*}$, with respect to the split quadratic form $q(a \oplus b)=b a$, which is invariant under the action of $\mathbb{Z} / p \mathbb{Z}$.

Fix the homomorphism $\rho$. Write $\boldsymbol{M}=\rho(\epsilon)$, and let $\alpha$ and $\gamma$ be as in (1). Denote the chosen basis of $A$ by $e_{1}, e_{2}$. This gives a basis $e_{1}, e_{2}, e_{1}^{*}, e_{2}^{*}$ for $A \oplus A^{*}$. Because $\gamma^{-1} \gamma^{*}$ and $\alpha$ have the same centralizer, Lemma 3.5 and eq. (6) imply that we may assume

$$
\gamma=\left(\begin{array}{cc}
-1 & -\left(\lambda+\lambda^{-1}+1\right) \\
1 & -1
\end{array}\right)
$$

Thus,

$$
\boldsymbol{M}=\left(\begin{array}{cccc}
\frac{a\left(\lambda^{2}+\lambda+1\right)}{\lambda^{2}+\lambda} & \frac{a}{\lambda+1} & -\frac{\lambda}{(\lambda+1)^{2}} & -\frac{\lambda}{(\lambda+1)^{2}} \\
-\frac{a}{\lambda+1} & \frac{a}{\lambda+1} & \frac{\lambda^{2}+\lambda+1}{(\lambda+1)^{2}} & -\frac{\lambda}{(\lambda+1)^{2}} \\
-1 & -\frac{\left(\lambda^{2}+\lambda+1\right)}{\lambda} & 0 & 0 \\
1 & -1 & 0 & 0
\end{array}\right) .
$$

For any element or subspace $a$ of $A \oplus A^{*}$, denote by $\pi a$ the projection of $a$ onto $A$. Fix some Lagrangian subspace $L$. We consider three cases.

Case 1: $\pi L$ has dimension 0. It follows that $L=A^{*}$, and by inspection, such Lagrangian subspaces are never invariant subspaces of the action of $\mathbb{Z} / p \mathbb{Z}$ by the homomorphism $\rho$.

Case 2: $\pi L$ has dimension 1. In this case, we prove that there is an invariant Lagrangian subspace if and only if $\lambda \in \mathbb{F}_{q}$.

First we will show the "only if" part. Since $\pi L$ has dimension 1, there is some vector $v \neq 0 \in \pi L$. We claim that $v \in L$. To see this, let $v^{\prime}$ be a lift of $v$ to $L$. Write 
$v^{\prime}=v+w$. Since $v+w \in L$, it suffices to show that $w \in L$. Take $w^{\prime} \in L$, such that $w^{\prime} \notin\left\langle v^{\prime}\right\rangle$. Since $\pi L$ has dimension 1 , we have that $\pi w^{\prime}=\lambda v$. Consider the vector $w^{\prime}-\lambda v^{\prime}$. We have $\pi\left(w^{\prime}-\lambda v^{\prime}\right)=0$, so $w^{\prime}-\lambda v^{\prime} \in A^{*}$. In view of $w^{\prime}-\lambda v^{\prime} \in L$ we have that

$0=q\left(\left(w^{\prime}-\lambda v^{\prime}\right)+v^{\prime}\right)=q\left(\left(w^{\prime}-\lambda v^{\prime}\right)+v+w\right)=\left(\left(w^{\prime}-\lambda v^{\prime}\right)+w\right)(v)=\left(w^{\prime}-\lambda v^{\prime}\right)(v)$,

since $w(v)=q\left(v^{\prime}\right)=0$. But from $w(v)=0$, it follows that $w \in\left\langle w^{\prime}-\lambda v^{\prime}\right\rangle \subset L$, since the subspace of $A^{*}$ which evaluates to 0 on a non-zero vector in $A$ is onedimensional. Therefore, $v \in L$. It follows that $\boldsymbol{M} v \in L$, and therefore that $\alpha v=$ $\pi(\boldsymbol{M} v) \in \pi L=\langle v\rangle$. Thus, $v$ is an eigenvector of $\alpha$. It follows that an eigenvalue of $\alpha$ lies in $\mathbb{F}_{q}$. Since the eigenvalues of $\alpha$ are $-\lambda$ and $-\lambda^{-1}$, we have that $\lambda \in \mathbb{F}_{q}$.

In order to see the "if" part, consider $L=\langle v, w\rangle$, where

$$
v=(1,-\lambda, 0,0), \quad w=(0,0, \lambda, 1) .
$$

Since $v \in A, w \in A^{*}$, and $w(v)=0$, it is clear that $L$ is a Lagrangian subspace. Thus, it suffices to show that $L$ is invariant under the action of $\mathbb{Z} / p \mathbb{Z}$, or that $\boldsymbol{M} v, \boldsymbol{M} w \in$ $\langle v, w\rangle$. By (7),

$$
\boldsymbol{M} v=\frac{a}{\lambda} v+(\lambda+1) w \quad \text { and } \quad \boldsymbol{M} w=-\frac{1}{\lambda^{-1}+1} v
$$

Case 3: $\pi L$ has dimension 2. In this case, we prove that there is no invariant Lagrangian subspace if $\lambda \notin \mathbb{F}_{q}$. Assume to the contrary. Since $\pi L$ has dimension $2, e_{1}, e_{2} \in \pi L$. Let $v$ be an arbitrary lift of $e_{1}$ to $L$, and $w$ be an arbitrary lift of $e_{2}$ to $L$. Clearly, we have $L=\langle v, w\rangle$. Since $q(v)=0$ and $\pi v=e_{1}, v$ must have the form $(1,0,0, s)$ for some $s \in \mathbb{F}_{q}$. Similarly, $w$ must have the form $\left(0,1, s^{\prime}, 0\right)$. Since $q(v+w)=0$, we have that $s^{\prime}=-s$. In other words, our Lagrangian subspace would have to be the span of two vectors in the form $v=(1,0,0, s), w=(0,1,-s, 0)$. We have $\boldsymbol{M} v \in\langle v, w\rangle$. Now we explicitly compute $\boldsymbol{M} v=(\kappa, \tau,-1,1)$, where

$$
\kappa=\frac{a(\lambda+1)\left(\lambda^{2}+\lambda+1\right)-s \lambda^{2}}{\lambda(\lambda+1)^{2}}, \quad \tau=-\frac{s \lambda+a(\lambda+1)}{(\lambda+1)^{2}} .
$$

Since $\boldsymbol{M} v \in\langle v, w\rangle$, we have that there exists $c_{v}, c_{w} \in \mathbb{F}_{q}$ with $c_{v} v+c_{w} w-\boldsymbol{M} v=0$. Since $\pi\left(c_{v} v+c_{w} w\right)=\left(\begin{array}{c}c_{v} \\ c_{w}\end{array}\right)$, we know that $c_{v}=\kappa$ and $c_{w}=\tau$. It follows that $0=\kappa v+\tau w-\boldsymbol{M} v=(0,0,1-\tau s, \kappa s-1)$. As

$$
1-\tau s=\frac{\left(s \zeta_{2}+\zeta_{1} \zeta_{2}+1\right)\left(s \zeta_{1}+\zeta_{1} \zeta_{2}+1\right)}{\zeta_{1}^{2} \zeta_{2}^{2}+2 \zeta_{1} \zeta_{2}+1}
$$

we deduce that

$$
\left(s \zeta_{2}+\zeta_{1} \zeta_{2}+1\right)\left(s \zeta_{1}+\zeta_{1} \zeta_{2}+1\right)=0
$$


Without loss of generality we may assume that $s=-\zeta_{1}-\zeta_{2}^{-1}$. Then

$$
0=\kappa s-1=-\frac{\left(\zeta_{1}+\zeta_{2}\right)\left(\zeta_{1} \zeta_{2}+1\right)^{2}}{\zeta_{1} \zeta_{2}^{2}} .
$$

But this is impossible, as $\zeta_{1}, \zeta_{2}$ are $p$-th roots of unity, $p$ is odd, $\zeta_{1} \zeta_{2} \notin \mathbb{F}_{q}$, and $\zeta_{1} \neq \zeta_{2}$. (The last two assumptions are needed only when $q=2$.)

At this point we can count the number of non-group-theoretical categories of dimension $p q^{2}$ up to grading-equivalence, and up to general equivalence. If $p$ does not divide $q^{2}-1$, then all categorifications are pointed. If $p$ divides $q-1$, then all $p$-th roots of unity lie in $\mathbb{F}_{q}$. Therefore, non-group-theoretical categorifications occur only when $p$ divides $q+1$; we have one such categorification for each pair $\left\{\zeta_{1}, \zeta_{2}\right\}$ such that $\zeta_{1} \zeta_{2} \neq 1$.

To account for grading-equivalences, we first compute the number $\frac{(p-1)(p-3)}{4}$ of pairs $\left\{\zeta_{1}, \zeta_{2}\right\}$ with $\zeta_{i} \neq 1$ up to equivalence $\left\{\zeta_{1}, \zeta_{2}\right\} \sim\left\{\zeta_{1}^{-1}, \zeta_{2}^{-1}\right\}$. To this we add the number $\frac{p-1}{2}$ of pairs $\{1, \zeta\}$ up to equivalence $\{1, \zeta\} \sim\left\{1, \zeta^{-1}\right\}$, for a total of $\frac{(p-1)^{2}}{4}$ non-group-theoretical categories up to grading-equivalence.

To account for general equivalences, consider the action of Aut $\mathbb{Z} / p \mathbb{Z}$ on our categories. An element $g \in($ Aut $\mathbb{Z} / p \mathbb{Z})$ acts by multiplication by $g^{-2}$ on $\mathrm{H}^{3}\left(\mathbb{Z} / p \mathbb{Z}, \mathbb{C}^{*}\right)$, and sends $\left(\zeta_{1}, \zeta_{2}\right) \rightarrow\left(\zeta_{1}^{g}, \zeta_{2}^{g}\right)$. There are three orbits on $\mathrm{H}^{3}\left(\mathbb{Z} / p \mathbb{Z}, \mathbb{C}^{*}\right)$ under this action: the quadratic non-residues, the quadratic residues, and 0 in an orbit by itself. The stabilizer of the first two orbits is \pm 1 , which then acts on the pairs $\left\{\zeta_{1}, \zeta_{2}\right\}$ as in the graded case, giving $\frac{(p-1)^{2}}{4}$ categorifications each, for $\frac{(p-1)^{2}}{2}$ together. The $\{0\}$ orbit in $\mathrm{H}^{3}\left(\mathbb{Z} / p \mathbb{Z}, \mathbb{C}^{*}\right)$ yields two types of orbits on the set of pairs $\left\{\zeta_{1}, \zeta_{2}\right\}$. Clearly Aut $\mathbb{Z} / p Z$ acts transitively on pairs $\{1, \zeta\}$. So suppose that $\zeta_{2}=\zeta_{1}^{k}$. Then the set $\left\{k, k^{-1}\right\}$ determines the orbit, and the number of such sets which do not contain 0 , \pm 1 is $\frac{p-3}{2}$. This gives a total of $\frac{p^{2}-p}{2}$ categorifications.

\section{Proof of Corollary 1.2}

By Lemma 3.6, all categories of dimension $p q^{2}$ without a faithful $\mathbb{Z} / p \mathbb{Z}$-grading are group-theoretical. Let us suppose that $\ell$ of dimension $p q^{2}$ is faithfully $\mathbb{Z} / p \mathbb{Z}$ graded and is the category of representations of some semi-simple Hopf algebra $H$, and demonstrate that $\mathcal{C}$ is group-theoretical.

The faithful $\mathbb{Z} / p \mathbb{Z}$-grading on $\mathcal{C}$ induces a faithful $\mathbb{Z} / p \mathbb{Z}$-grading on $H^{*}$ as follows. Since $\mathcal{C}$ is faithfully $\mathbb{Z} / p \mathbb{Z}$-graded, there exists a central group-like element $c \in H$ such that $c^{p}=1$, defining the grading. This element defines the decomposition $H^{*}=\bigoplus_{k} H_{k}^{*}$, where $H_{k}^{*}=\left\{f \in H^{*} \mid f(c x)=\zeta^{k} f(x)\right\}$ and $\zeta=e^{\frac{2 \pi i}{p}}$. Clearly $H_{k}^{*} \neq 0$ for all $k$. 
We consider the sub-algebra $H_{0}^{*}$ of $H^{*}$, and we let $\mathscr{D}$ denote the category of $H_{0}^{*}$-bimodules in $\mathcal{C} ; \mathcal{D}$ is Morita equivalent to $\mathcal{C}$. Because $H_{0}^{*} \in \mathcal{C}_{0}, \mathscr{D}$ is also $\mathbb{Z} / p \mathbb{Z}$-graded, and we have $\left|\mathscr{D}_{0}\right|=q^{2}$, so $\mathscr{D}_{0}$ is pointed. Furthermore, $H^{*}$ is an algebra in $\mathscr{D}$, whose 0 -component is $H_{0}^{*}$, the unit in $\mathscr{D}$.

Claim 5.1. The multiplication map $\mu: H_{k}^{*} \otimes_{H_{0}^{*}} H_{l}^{*} \rightarrow H_{k+l}^{*}$ is an isomorphism for all $k$ and $l$.

Proof. We claim first that the map

$$
\Delta_{l}=\left(\pi_{l} \otimes \mathrm{Id}\right) \circ \Delta: H \rightarrow H /\left(c-\zeta^{l}\right) \otimes H
$$

is injective. Indeed, suppose that $a \in H$ such that $\Delta_{l}(a)=0$. Then for all $V \in \mathscr{C}_{l}$, $U \in \mathcal{C}$, we have that $\left.a\right|_{V \otimes U}=0$. Taking $U=H$, we have $V \otimes H \cong(\operatorname{dim} V) H$, so $a$ must be zero. By duality,

$$
\mu: H^{*} \otimes_{H_{0}^{*}} H_{l}^{*} \rightarrow H^{*}
$$

is surjective. By the Nichols-Zoeller theorem [NZ], $H^{*}$ is free over $H_{0}^{*}$ of rank $p$. Therefore, the left-hand side of (8) has dimension $p \cdot \operatorname{dim} H_{l}^{*}=\operatorname{dim} H^{*}$. Thus (8) is an isomorphism. Restricting to the graded components yields the claim.

The claim implies that each $H_{k}^{*}$ is an invertible object in $\mathscr{D}$, and so in particular there are invertible objects in each $\mathscr{D}_{k}$, in addition to the $q^{2}$ invertible objects in $\mathscr{D}_{0}$. As the number of invertible objects must divide the overall dimension $p q^{2}$ of $\mathscr{D}$, we conclude that $\mathscr{D}$ is pointed.

\section{Categorifications of $R_{3, A}$}

Lemma 6.1. If $|A|$ is coprime to 3 , $\mathbb{Z} / 3 \mathbb{Z}$-graded categories $C$ with trivial component $\operatorname{Vec}_{A}$ such that $\mathcal{C}_{\epsilon}$ contains a single simple object are all categorifications of $R_{3, A}$, and are, up to grading-equivalence, parameterized by pairs $(\xi, \gamma)$, where $\xi$ is an element of $\mathrm{H}^{3}\left(\mathbb{Z} / 3 \mathbb{Z}, \mathbb{C}^{*}\right) \simeq \mathbb{Z} / 3 \mathbb{Z}$, and $\gamma$ is a map $A \rightarrow A^{*}$ such that $\gamma^{*} \gamma^{-1} \gamma^{*}$ is skew-symmetric. If our equivalence is not required to preserve grading, we must additionally identify $\gamma$ with $\gamma^{*}$.

Proof. Clearly, categorifications of $R_{3, A}$ are $\mathbb{Z} / 3 \mathbb{Z}$-graded categories $\mathcal{C}$ with trivial component $\operatorname{Vec}_{A}$ such that $\ell_{\epsilon}$ contains a single simple object. To see the reverse inclusion, recall that $\mathcal{C}_{g}$ is contains a unique simple object if and only if $\rho(g)$ has its upper right entry an isomorphism. Thus, it suffices to show that $\rho(g)$ has its upper right entry an isomorphism if, and only if, $\rho\left(g^{-1}\right)$ does. But this is clear because $\rho$ is a homomorphism into the split orthogonal group, so the upper right entry of $\rho(g)$ is the 
dual of the upper right entry of $\rho\left(g^{-1}\right)$. Therefore, by Theorem 3.2, $\mathcal{C}$ is determined up to grading-equivalence by an element of $\mathrm{H}^{3}\left(\mathbb{Z} / 3 \mathbb{Z}, \mathbb{C}^{*}\right) \simeq \mathbb{Z} / 3 \mathbb{Z}$, together with a map $\alpha: A \rightarrow A$, and an isomorphism $\gamma: A \rightarrow A^{*}$, satisfying the relations $\gamma^{*} \alpha$ is skew-symmetric and (1). Write $x$ for $\gamma^{-1} \gamma^{*}$. To solve equation (1), we explicitly compute:

$$
\left(\begin{array}{cc}
\mathrm{Id} & 0 \\
0 & \mathrm{Id}
\end{array}\right)=\left(\begin{array}{cc}
\alpha & \mathrm{Id} \\
x^{-1} & 0
\end{array}\right)^{3}=\left(\begin{array}{cc}
\alpha^{3}+x^{-1} \alpha+\alpha x^{-1} & \alpha^{2}+x^{-1} \\
x^{-1} \alpha^{2}+x^{-2} & x^{-1} \alpha
\end{array}\right) .
$$

In particular, $\alpha=x$. The condition that $\gamma^{*} \alpha$ is skew-symmetric then becomes that $\gamma^{*} \gamma^{-1} \gamma^{*}$ is skew-symmetric, from which it follows that $\left(\gamma^{-1} \gamma^{*}\right)^{3}=-$ Id. When $\alpha=x$, and $x^{3}=-\mathrm{Id}$, it is not hard to check that (1) is satisfied.

In other words, the conditions on $(\alpha, \gamma)$ given by Theorem 3.2 are equivalent to $\alpha=\gamma^{-1} \gamma^{*}$, and $\gamma^{*} \gamma^{-1} \gamma^{*}$ skew-symmetric. Therefore, the choice of maps $\alpha$ and $\gamma$ is equivalent to the choice of a single map $\gamma$ such that $\gamma^{*} \gamma^{-1} \gamma^{*}$ is skew-symmetric.

Finally, in the case where we do not require that our equivalence preserves grading, we must figure out what happens under the action of Aut $\mathbb{Z} / 3 \mathbb{Z}$. In order to do this, we must consider what happens to $\gamma$ under the transformation $\boldsymbol{M} \rightarrow \boldsymbol{M}^{-1}=\boldsymbol{M}^{*}$. In our case,

$$
\boldsymbol{M}=\left(\begin{array}{cc}
\gamma^{-1} \gamma^{*} & \gamma^{*-1} \\
\gamma & 0
\end{array}\right), \quad \boldsymbol{M}^{*}=\left(\begin{array}{cc}
0 & \gamma^{-1} \\
\gamma^{*} & \gamma \gamma^{-1 *}
\end{array}\right)
$$

We find that

$$
\boldsymbol{M}^{*} \sim\left(\begin{array}{cc}
1 & 0 \\
\gamma^{*} \gamma^{-1} \gamma^{*} & 1
\end{array}\right)\left(\begin{array}{cc}
0 & \gamma^{-1} \\
\gamma^{*} & \gamma \gamma^{-1 *}
\end{array}\right)\left(\begin{array}{cc}
1 & 0 \\
-\gamma^{*} \gamma^{-1} \gamma^{*} & 1
\end{array}\right)=\left(\begin{array}{cc}
\gamma^{-1 *} \gamma & \gamma^{-1} \\
\gamma^{*} & 0
\end{array}\right),
$$

which is the same as the matrix $\boldsymbol{M}$ with $\gamma$ replaced with $\gamma^{*}$.

Lemma 6.2. Let $q \neq 3$, $A$ be an abelian q-group, and $\gamma$ a non-degenerate map $A \rightarrow A^{*}$ such that $\gamma^{*} \gamma^{-1} \gamma^{*}$ is skew-symmetric. Then $A$ may be decomposed as $\bigoplus_{i}\left(C_{i} \oplus C_{i}\right)$, for cyclic groups $C_{i}$, where the $C_{i} \oplus C_{i}$ are mutually orthogonal with respect to $\gamma$, and on each component $C_{i} \oplus C_{i}$, either $\gamma$ is skew-symmetric, or $\left(\gamma^{-1} \gamma^{*}\right)^{2}=\gamma^{-1} \gamma^{*}-$ Id.

Proof. Write $x=\gamma^{-1} \gamma^{*}$. Since $\gamma^{*} \gamma^{-1} \gamma^{*}$ is skew-symmetric, it follows that $x^{3}=$ - Id. Write $A^{\prime}=\operatorname{Ker}(x+\mathrm{Id})$ and $A^{\prime \prime}=\operatorname{Im}(x+\mathrm{Id})$.

First, we claim that $\operatorname{Ker}(x+\mathrm{Id})=\operatorname{Ker}(x+\mathrm{Id})^{2}$. Observe that if $(x+\mathrm{Id})^{2} g=0$, then, since $x^{3}=-\mathrm{Id}, 0=(x-2 \mathrm{Id})(x+\mathrm{Id})^{2} g=\left(x^{3}-3 x-2 \mathrm{Id}\right) g=-3(x+\mathrm{Id}) g$. Since $q \neq 3$, multiplication by -3 is invertible on $A$, and therefore $(x+\mathrm{Id}) g=0$.

It follows that $A=A^{\prime} \oplus A^{\prime \prime}$. Obviously, $x$ restricts to each component. It is clear that on $A^{\prime}, x$ is - Id. Since on $A^{\prime \prime}, x+\mathrm{Id}$ is invertible, and $0=x^{3}+\mathrm{Id}=$ $(x+\mathrm{Id})\left(x^{2}-x+\mathrm{Id}\right)$, we have that $x^{2}=x-\operatorname{Id}$ on $A^{\prime \prime}$. Now we claim that $\gamma$ 
restricts to each component. Since $\gamma: A \rightarrow A^{*}$, restricting to $\operatorname{Im}(x+\mathrm{Id})$ means that $\gamma: \operatorname{Im}(x+\operatorname{Id}) \rightarrow \operatorname{Im}(x+\operatorname{Id})^{*}$, and restricting to $\operatorname{Ker}(x+\mathrm{Id})$ means that $\gamma: \operatorname{Ker}(x+\mathrm{Id}) \rightarrow \operatorname{Ker}(x+\mathrm{Id})^{*}$. The first follows from the fact that $\gamma(x+\mathrm{Id})=$ $(x+\mathrm{Id})^{*} \gamma^{*}$, and the second follows from $\left(\gamma-\gamma^{*}\right)(x+\mathrm{Id})=(x+\mathrm{Id})^{*} \gamma$.

We have shown that $A=A^{\prime} \oplus A^{\prime \prime}$ where $x$ and $\gamma$ restrict to $A^{\prime}$ and $A^{\prime \prime}, x$ is - Id on $A^{\prime}$, and $x^{2}=x-\operatorname{Id}$ on $A^{\prime \prime}$. Denote by $n$ the unique natural number so that $q^{n} A^{\prime \prime}=0$, but $q^{n-1} A^{\prime \prime} \neq 0$.

We will show that $A^{\prime \prime}$ decomposes as a direct sum $\bigoplus_{i}\left(C_{i} \oplus C_{i}\right)$ which respects $\gamma$, by strong induction on $\left|A^{\prime \prime}\right|$. The base case, where $\left|A^{\prime \prime}\right|=1$, is trivial.

In order to do the inductive step, first suppose that $q^{n-1} \gamma(g, g)=0$ for any $g$ such that there does not exist a $g^{\prime}$ where $g=q g^{\prime}$. Since any element of $A^{\prime \prime}$ is a multiple of some such $g$, we would have that $q^{n-1} \gamma(g, g)=0$ for any $g \in A^{\prime \prime}$. From this, we would have that $q^{n-1} \gamma^{*}=-q^{n-1} \gamma$; therefore, by the definition of $x$, we would have that $\gamma^{*}=\gamma x$ and $q^{n-1} x=-q^{n-1}$ Id. Because $x^{2}=x-$ Id, $q^{n-1} \mathrm{Id}=-\left(q^{n-1} x\right)=x\left(-q^{n-1} \mathrm{Id}\right)=q^{n-1} x^{2}=q^{n-1} x-q^{n-1} \mathrm{Id}=-2 q^{n-1} \mathrm{Id}$. Therefore, $3 q^{n-1} \mathrm{Id}=0$. Since $q \neq 3$, we would have $q^{n-1} \mathrm{Id}=0$, a contradiction.

Thus, we have that there is some $g$ such that there does not exist a $g^{\prime}$ with $g=q g^{\prime}$ and such that $q^{n-1} \gamma(g, g) \neq 0$. Write $B=\operatorname{Ker} \gamma g \cap \operatorname{Ker} \gamma^{*} g$. We claim that $A^{\prime \prime}=B \oplus\langle g\rangle \oplus\langle x g\rangle$. To verify this, it suffices to show that the map $(a, b) \rightarrow\left(\gamma(g, a g+b x g), \gamma^{*}(g, a g+b x g)\right)$ which maps $\mathbb{Z} / q^{n} \mathbb{Z} \times \mathbb{Z} / q^{n} \mathbb{Z} \rightarrow$ $\left(\bigcup_{g \in A^{*}} \operatorname{Im} g\right)^{2}$ is an isomorphism. This is clear from the explicit computation that $\left(\gamma(g, a g+b x g), \gamma^{*}(g, a g+b x g)\right)=((a+b) s, a s)$, where $s=\gamma(g, g)$ is a generator of $\bigcup_{g \in A^{*}} \operatorname{Im} g$. Since $\operatorname{Ker} \gamma x g=\operatorname{Ker} \gamma^{*} g$ and $\operatorname{Ker} \gamma^{*} x g=\operatorname{Ker}\left(\gamma^{*}-\gamma\right) g$, it is clear that $B$ is orthogonal to $\langle g\rangle \oplus\langle x g\rangle$. Applying the inductive hypothesis to $B$ completes the proof.

The proof that $A^{\prime}$ decomposes as a direct sum $\bigoplus_{i}\left(C_{i} \oplus C_{i}\right)$ which respects $\gamma$, under the assumption that $\gamma$ is skew-symmetric, is the standard proof that every skewform has a symplectic basis over a vector space, where instead of splitting off $\left\langle v, v^{\prime}\right\rangle$ so that $\gamma\left(v, v^{\prime}\right) \neq 0$, we split off $\left\langle g, g^{\prime}\right\rangle$ such that the order of the cyclic subgroup generated by $g$ is $q^{n}$, and so that $q^{n-1} \gamma\left(g, g^{\prime}\right) \neq 0$.

\section{Proof of Theorem 1.4}

By Lemma 6.1, the categorifications are in one to one correspondence with an element of $\mathrm{H}^{3}\left(\mathbb{Z} / 3 \mathbb{Z}, \mathbb{C}^{*}\right) \simeq \mathbb{Z} / 3 \mathbb{Z}$ together with a map $\gamma: A \rightarrow A^{*}$ satisfying $\left(\gamma^{-1} \gamma^{*}\right)^{3}=$ - Id. In order to classify such forms up to equivalence, it suffices to classify such forms on the $q$-parts of $A$ for each prime $q$. By our Lemmas 3.4, 3.5, and 6.2, there are $\prod\left(a_{i} / 2+1\right)$ choices for $\gamma$, as on each $C_{i} \oplus C_{i}$ there are two choices, depending on whether or not $\gamma$ is skew-symmetric. Since there are three choices for the element of $\mathrm{H}^{3}\left(\mathbb{Z} / 3 \mathbb{Z}, \mathbb{C}^{*}\right) \simeq \mathbb{Z} / 3 \mathbb{Z}$, the statement of this corollary follows, 
provided that we can show that $\operatorname{Aut}(\mathbb{Z} / 3 \mathbb{Z})$ acts trivially on $H^{3}\left(\mathbb{Z} / 3 \mathbb{Z}, \mathbb{C}^{*}\right)$ and our solutions for $\gamma$. To see that it acts trivially on the cohomology group, recall that $\mathrm{H}^{3}\left(\mathbb{Z} / 3 \mathbb{Z}, \mathbb{C}^{*}\right)=(\mathbb{Z} / 3 \mathbb{Z})^{\otimes(-2)}$. To see that it acts trivially on our solutions, observe that $\gamma$ being skew-symmetric is the same as $\gamma^{*}$ being skew-symmetric. As $\gamma$ is determined by on how many of each type of $C_{i} \oplus C_{i}$ it is skew-symmetric, $\gamma$ and $\gamma^{*}$ are equivalent. Thus, the statement of the theorem follows.

\section{References}

[DGNO] V. Drinfeld, S. Gelaki, D. Nikshych, and V. Ostrik, Group-theoretical properties of nilpotent modular categories. Preprint 2007. arXiv:0704.0195v2

[EGO] P. Etingof, S. Gelaki, and V. Ostrik, Classification of fusion categories of dimension pq. Internat. Math. Res. Notices 2004 (2004), 3041-3056. Zbl 1063.18005 MR 2098028

[ENO1] P. Etingof, D. Nikshych, and V. Ostrik, On fusion categories. Ann. of Math. (2) 162 (2005), 581-642. Zbl 1125.16025 MR 2183279

[ENO2] P. Etingof, D. Nikshych, and V. Ostrik. Weakly group-theoretical and solvable fusion categories. Preprint 2008. arXiv:0809.3031v1

[ENO3] P. Etingof, D. Nikshych, and V. Ostrik. Fusion categories and homotopy theory. Preprint in preparation.

[GNN] S. Gelaki, D. Naidu, and D. Nikshych. Centers of nilpotent fusion categories. Preprint in preparation.

[NZ] W. D. Nichols and M. B. Zoeller, A Hopf algebra freeness theorem. Amer. J. Math. 111 (1989), 381-385. Zbl 0672.16006 MR 987762

[O1] V. Ostrik, Module categories, weak Hopf algebras and modular invariants. Transform. Groups 8 (2003), 177-206. Zbl 1044.18004 MR 1976459

[O2] V. Ostrik, Module categories over the Drinfeld double of a finite group. Internat. Math. Res. Notes 2003 (2003), 1507-1520. Zbl 1044.18005 MR 1976233

[O3] V. Ostrik, Fusion categories of rank 2. Math. Res. Lett. 10 (2003), 177-183. Zbl 1040.18003 MR 1981895

[TY] D. Tambara and S. Yamagami, Tensor categories with fusion rules of self-duality for finite abelian groups. J. Algebra 209 (1998), 692-707.Zbl 0923.46052 MR 1659954

Received April 14, 2009

D. Jordan, Department of Mathematics, Massachusetts Institute of Technology, Cambridge, MA 02139, U.S.A.

E-mail: djordan@math.mit.edu

E. Larson, South Eugene High School, 400 East 19th Avenue, Eugene, OR 97401, U.S.A. 